\title{
T-Cadherin Expression in the Epidermis and Adnexal Structures of Normal Skin
}

\author{
Stanislaw Buechner ${ }^{a}$ Paul Erne ${ }^{b}$ Therese J. Resink ${ }^{b}$ \\ ${ }^{a}$ Dermatology Private Office and Laboratory for Histologic Diagnostic, and \\ b Laboratory for Signal Transduction, Department of Biomedicine, Basel University and \\ Basel University Hospital, Basel, Switzerland
}

\section{Key Words}

T-cadherin $\cdot$ Immunohistochemistry $\cdot$ Epidermis $\cdot$ Skin appendages

\begin{abstract}
Background: T-cadherin is an atypical glycosylphosphatidylinositol-anchored member of the cadherin superfamily of adhesion molecules. The role of T-cadherin in biology of the skin is poorly understood. Expression of T-cadherin in basal keratinocytes and dermal blood vessels of the healthy epidermis has been demonstrated, but studies on expression in skin appendages are rare. Methods: We conducted an immunohistochemical analysis of T-cadherin expression in the epidermis and adnexal structures of normal skin. Results: T-cadherin expression is restricted to basal keratinocytes of the epidermis. The basal cell layer of sebaceous glands was T-cadherin positive, whereas sebocytes were negative. Within apocrine glands, only myoepithelial cells were T-cadherin positive. In contrast, both the secretory coils and excretory ducts of eccrine glands were T-cadherin positive. In terminal hair follicles, the outer root sheath layers strongly expressed T-cadherin throughout different regions of the follicle, with the strongest immunoreactivity at the bulge and suprabulbar regions. T-cadherin and CK15 stem cell marker similarly localized within the bulge and suprabulbar region. T-cadherin and CD34 stem cell marker similarly localized at the suprabulbar level. Conclusion: The specific patterns of T-cadherin expression in the epidermis and adnexal structures suggest an important guardian role in skin homeostasis.

\section{Introduction}

Cadherins are a superfamily of transmembrane proteins that mediate calcium-dependent intercellular adhesion [1]. The cadherin superfamily consists of classic cadherins and nonclassic cadherins, including desmosomal cadherins and the recently discovered protocad- 
herins [2]. Major cadherins in the skin include the adherens junction cadherin E-cadherin and the desmosomal cadherin desmoglein 1 ; these are expressed by virtually all epidermal keratinocytes and melanocytes and play important roles in maintaining proper cohesive arrangement of the epidermal cells [1,3].

A cadherin less well studied in the context of the skin is T-cadherin. T-cadherin is a unique cadherin superfamily member: it possesses the five extracellular cadherin domain repeat structures typical of the classical cadherins but lacks transmembrane and cytoplasmic domains and is instead anchored to the cell membrane via a glycosylphosphatidylinositol moiety [4]. T-cadherin is strongly expressed on epidermal basal layer keratinocytes of human [5-8] and murine [7] skin. Very little information exists regarding expression of T-cadherin in other structures of the skin. Its presence on the hair follicle, sebaceous glands, and eccrine glands has been reported $[5,6]$; however, details on cellular distribution and pattern of expression are imprecise. In order to gain insight into the potential functions of T-cadherin in the skin, we performed an extensive examination of T-cadherin expression and its distribution in the epidermis and adnexal structures of normal skin.

\section{Materials and Methods}

Ten normal skin specimens obtained from the samples of wide surgical resection of cutaneous tumors were used. Immunohistochemical studies were performed on 4 - $\mu$ m-thick sections cut from formalin-fixed paraffin-embedded tissue using a universal immunoperoxidase polymer detection system (N-Histofine Simple Stain MAX PO; Nichirei Biosciencies, Tokyo, Japan). Sections were deparaffinized in xylol and hydrated through grades of ethanol, and then heated in a citric acid buffer $\left(\mathrm{pH} \mathrm{6.0)}\right.$ at $95^{\circ} \mathrm{C}$ for $60 \mathrm{~min}$ for antigen retrieval. Endogenous peroxidase was blocked with $0.3 \%$ hydrogen peroxide for $10 \mathrm{~min}$. The primary antibodies used were goat anti-T-cadherin (1:300; cat. No. AF3264, R\&D Systems Europe Ltd., Abingdon, UK), monoclonal mouse anti-CD34 (undiluted [RTU-end], Novocastra), and rabbit anti-cytokeratin 15 (CK15) mAb EPR1614Y (1:300; cat. No. ab52816, Abcam, Lucerna-Chem AG, Lucerne, Switzerland). Sections were incubated with the primary antibodies at room temperature for $60 \mathrm{~min}$. Immunoreactivity was visualized by using the Histofine Simple Stain MAX PO reagent and diaminobenzidine tetrahydrochloride as the substrate. Sections were counterstained with Mayer's hematoxylin, dehydrated with grades of ethanol, cleared with xylene, and mounted with Tissue Tec coverslipping film. For negative controls, the primary antibody was omitted from the staining protocol. Immunohistochemical reactions on any given tissue sample and using any given antibody were run in duplicate. Stained sections were viewed using a Nikon Eclipse 50i microscope and images captured using a ColorView IIIu camera (Olympus).

\section{Results}

\section{T-Cadherin Expression in Normal Epidermis}

$\mathrm{T}$-cadherin was strongly expressed in the basal keratinocyte layer, with a typically global distribution over the cell membrane and pronounced expression at intercellular junctions and on the basal and apical sides of the epidermal basal cells (Fig. 1a). There was a weak cell body and/or nuclear expression of T-cadherin on occasional suprabasal keratinocytes (Fig. 1a). Except for isolated cells, there was no T-cadherin expression in the upper spinous layer of the epidermis. 

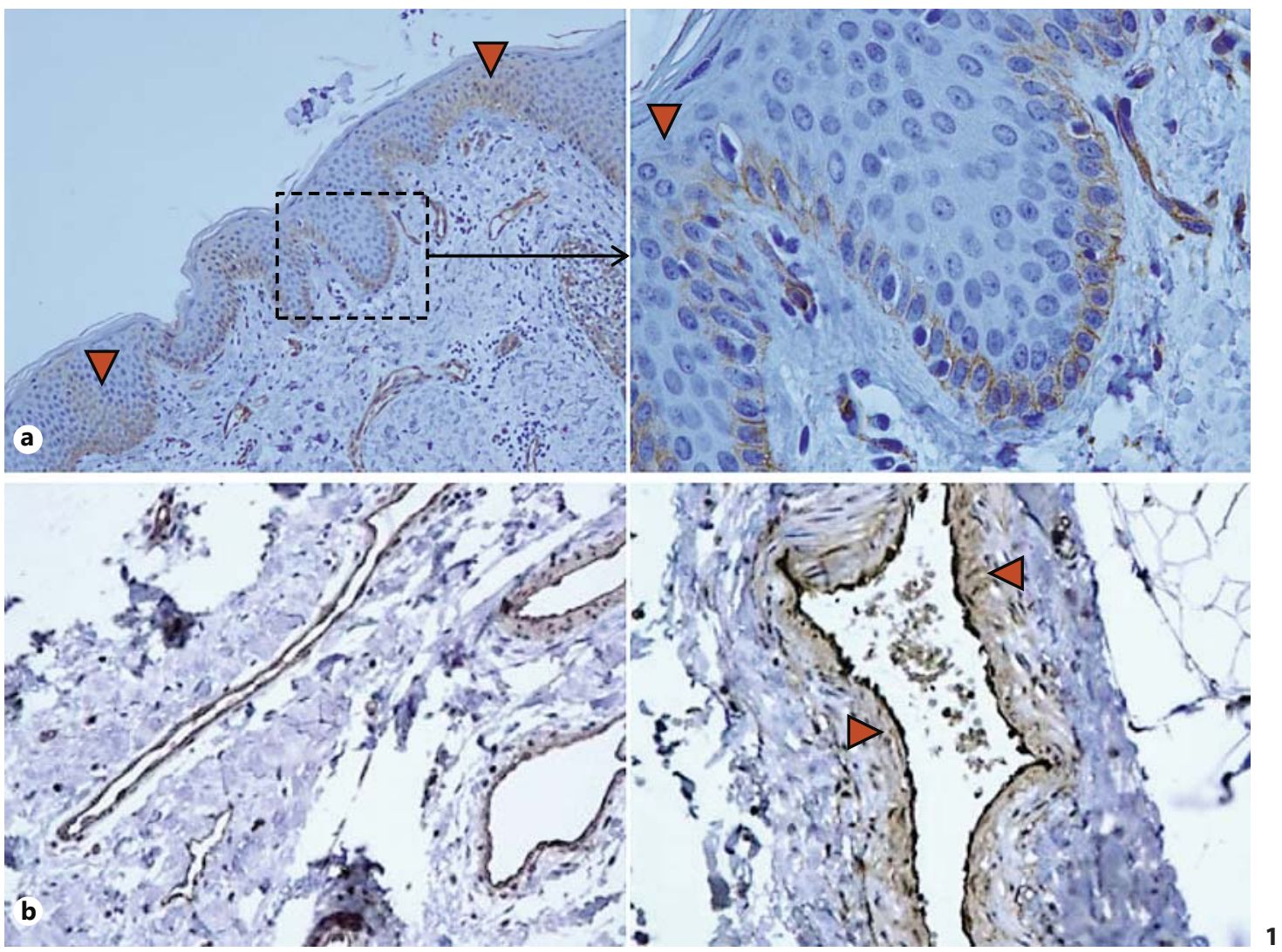

Fig. 1. T-cadherin expression in normal epidermis. Note the strong staining in the basal keratinocyte layer, with a typically global distribution over the cell body and pronounced expression on the basal side and intercellular contacts of the epidermal basal cells (a). Close-up showing loss of T-cadherin expression in the suprabasal layers of epidermis (arrowheads; a). Strongly positive expression of Tcadherin on endothelial cells of dermal blood vessels is evident $(\mathbf{a}, \mathbf{b})$. Note the less intense staining for T-cadherin within the subendothelial smooth muscle cell layers in arteries (arrowheads; b).

Fig. 2. T-cadherin expression in sebaceous glands. Positive staining for T-cadherin is demonstrated in the basal cell layer (arrowheads) of the sebaceous gland, whereas mature sebocytes in the central portion of the glands are negative for T-cadherin.

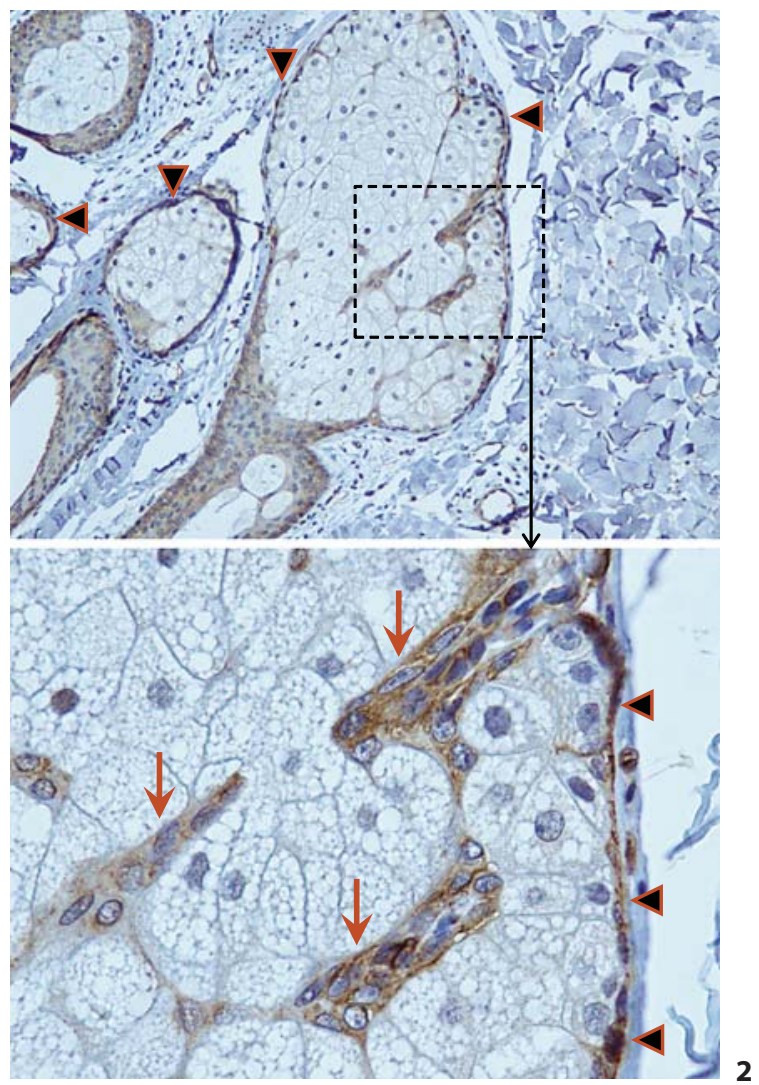




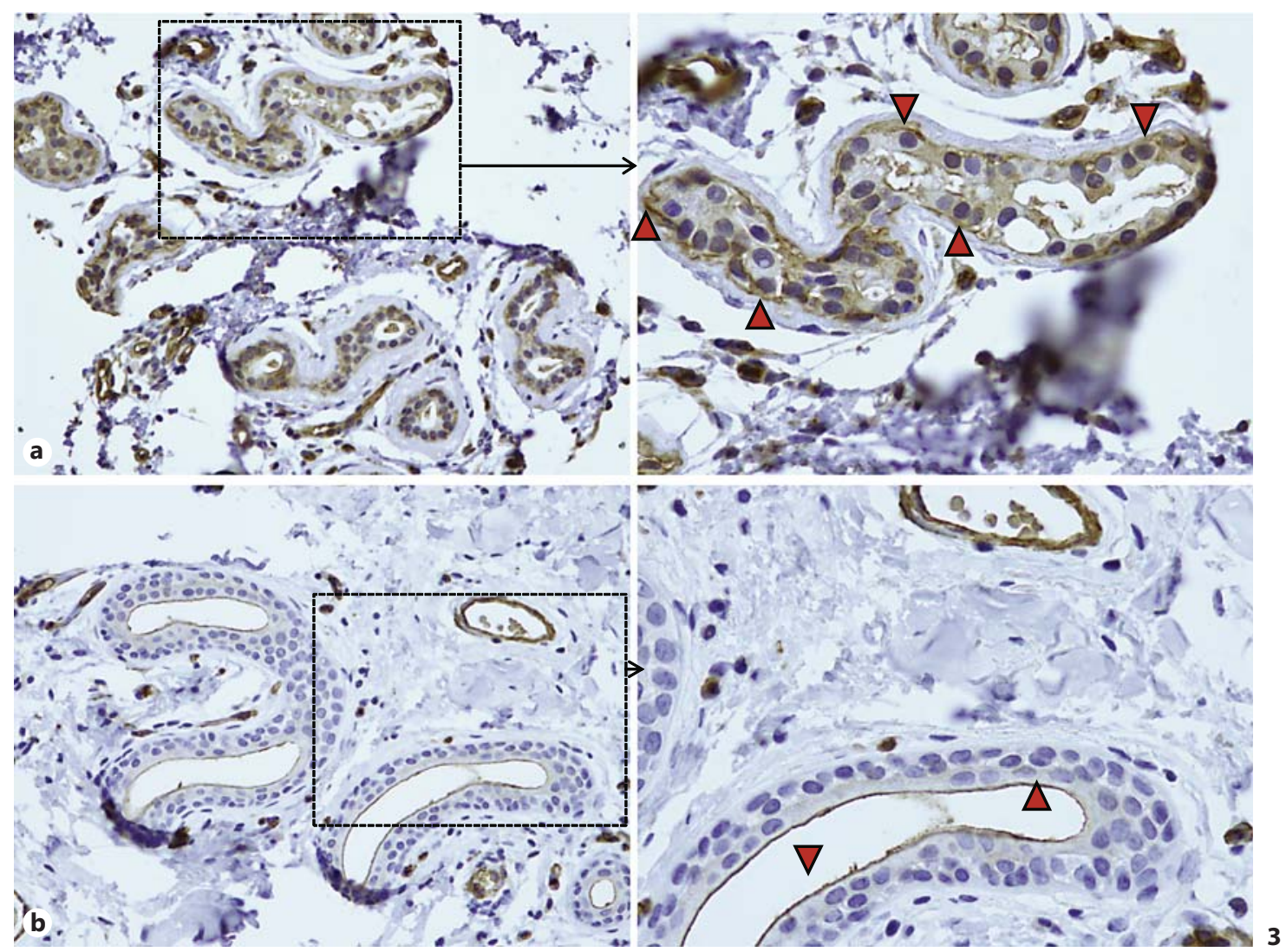

Fig. 3. T-cadherin expression in eccrine glands. In the normal eccrine glands, the secretory cells in the secretory coils of the glands (a) typically express $\mathrm{T}$-cadherin over the entire cell body. Note the myoepithelial cells of the secretory portion expressing T-cadherin (arrowheads). The inner layer of epithelial cells lining the excretory ducts of eccrine glands (b) exhibit pronounced luminal staining for T-cadherin (arrowheads).

Fig. 4. T-cadherin expression in apocrine glands. In the normal apocrine glands, only the peripheral myoepithelial cells show T-cadherin immunoreactivity (arrowheads).

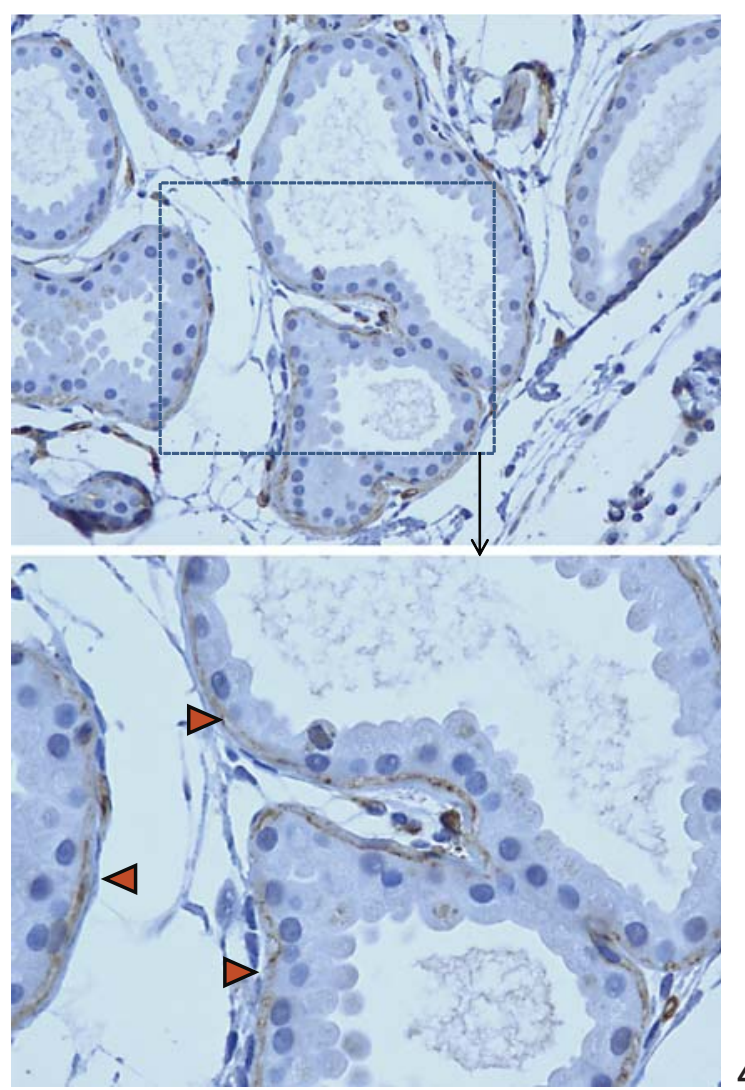


T-Cadherin Expression in the Dermal Blood Vessels

$\mathrm{T}$-cadherin was present on endothelial cells in all types of dermal blood vessels. T-cadherin staining was most prominent on the luminal side of endothelial cells. Subendothelial smooth muscle cells and pericytes also showed immunoreactivity for T-cadherin (Fig. 1b).

\section{T-Cadherin Expression in the Sebaceous, Eccrine, and Apocrine Glands}

Within sebaceous glands, moderately to strongly positive staining for T-cadherin was observed in the basal cell layer surrounding maturing sebaceous cells. The sebaceous cells were consistently negative for T-cadherin (Fig. 2).

In the eccrine glands, moderately to strongly positive cytoplasmic and membranous staining for T-cadherin was found in the majority of the single layer of cells within the secretory portion. T-cadherin was also strongly expressed on the outer myoepithelial cells surrounding the coiled gland (Fig. 3a). The eccrine ducts consisting of a double layer of cells exhibited a strong membranous staining on the inner layer of cuticular cells, although infrequently, a few cells with cytoplasmic staining were seen (Fig. 3b).

In contrast to the pattern of staining in eccrine glands, both the coiled secretory gland and excretory ducts of apocrine glands were consistently negative for T-cadherin, whereas the myoepithelial cell layer around the periphery of the glands demonstrated moderately strong staining for T-cadherin (Fig. 4).

\section{T-Cadherin Expression in the Hair Follicles}

In order to define the distribution for T-cadherin in terminal hair follicles, we used vertical consecutive sections of the hair follicles and compared the localization of T-cadherin with that of two usually used stem cell markers, CD34 and CK15. In terminal hair follicles, the basal cell layer of the epidermis and follicular infundibulum was strongly positive for T-cadherin and CK15 but negative for CD34. CD34 was strongly expressed in the endothelial cell and perifollicular spindle-shaped cells (Fig. 5a). A strong staining for T-cadherin was observed in the outer root sheath (ORS) layers within the isthmus of the follicle: the distribution was typically global with staining intensity being more prominent on the apical surface and at intercellular contacts of cells (Fig. 5b). The area of the ORS around the site where the arrector pili muscle attaches to the hair follicle (otherwise known as the bulge) demonstrated intense staining for T-cadherin (Fig. 5b). In contrast, the inner root sheath of the hair follicles was T-cadherin negative. Strong CK15 immunoreactivity was found in the ORS of the isthmus and with increasing staining intensity at the site of attachment of the arrector pili muscle at the bulge level (Fig. 5b). CD34 staining was not observed in the ORS within the level of the isthmus (Fig. 5b).

In the suprabulbar zone, the ORS layers exhibited strong staining for T-cadherin and CK15 (Fig. 6). CD34 was expressed in the most external layer of the ORS below the level of the isthmus, but was not found in the lower part of the suprabulbar ORS region (Fig. 6).

Within the thin ORS layers surrounding the bulb of the follicle, positive T-cadherin was expressed in most cells, whereas CK15 expression was more focal (Fig. 7). In the surrounding fibrous root sheath of the hair bulb and in the dermal papilla, isolated cells with immunoreactivity for T-cadherin were observed. CD34 immunoreactivity was absent in the hair bulb (Fig. 7).

\section{Discussion}

Expression of T-cadherin on keratinocytes and specific localization on the basal cell layer of the epidermis in normal skin was first reported by Zhou and colleagues [7] more than a decade ago. This finding was confirmed in a number of subsequent studies addressing 

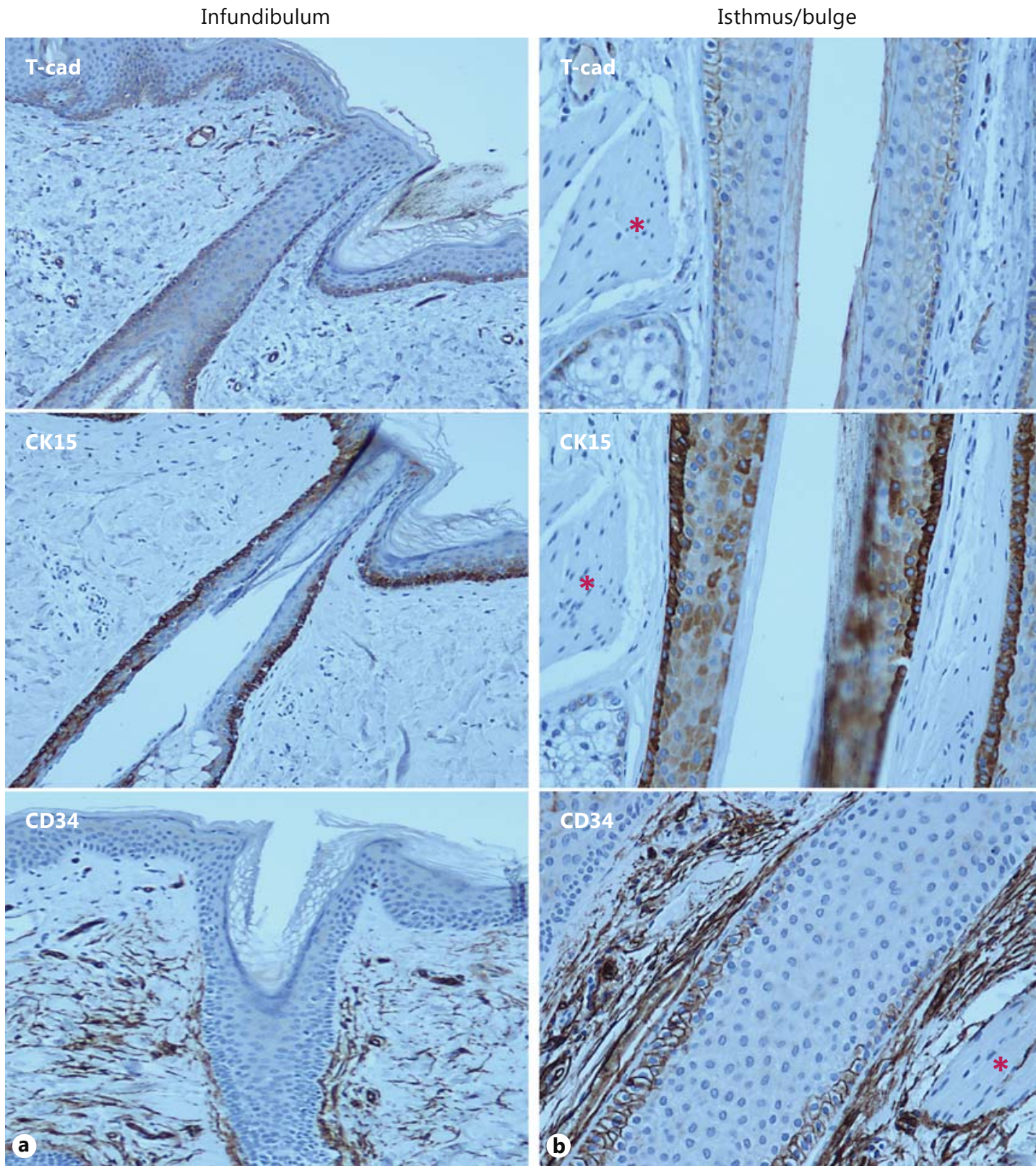

Fig. 5. Expression of T-cadherin, CK15, and CD34 in the infundibulum and isthmus in serial sections of terminal hair follicles. a The ORS layer of the hair follicle infundibulum demonstrates positive membranous staining for T-cadherin and CK15, whereas no staining for CD34 is present. b Note that T-cadherin immunoreactivity prominently marks ORS cells within the lower isthmus near the arrector pili muscle (indicated by asterisks) attachment site of the hair follicle known as the bulge. T-cadherin and CK15 stem cell marker similarly localized within the bulge and suprabulbar region. T-cadherin and CD34 stem cell marker similarly localized at the suprabulbar level.

expression of T-cadherin in a variety of keratinocytic disorders including psoriasis [9], basal cell carcinoma (BCC) $[5,8]$, actinic keratosis $[6,8]$, and squamous cell carcinoma (SCC) $[6,10]$. In this study, we demonstrate that $\mathrm{T}$-cadherin exhibits a precisely confined pattern of expression not only in the normal epidermis but also in adnexal structures of the skin, including the sebaceous, eccrine and apocrine glands and the hair follicle. 
Buechner et al.: T-Cadherin Expression in the Epidermis and Adnexal Structures of Normal Skin

Suprabulbar
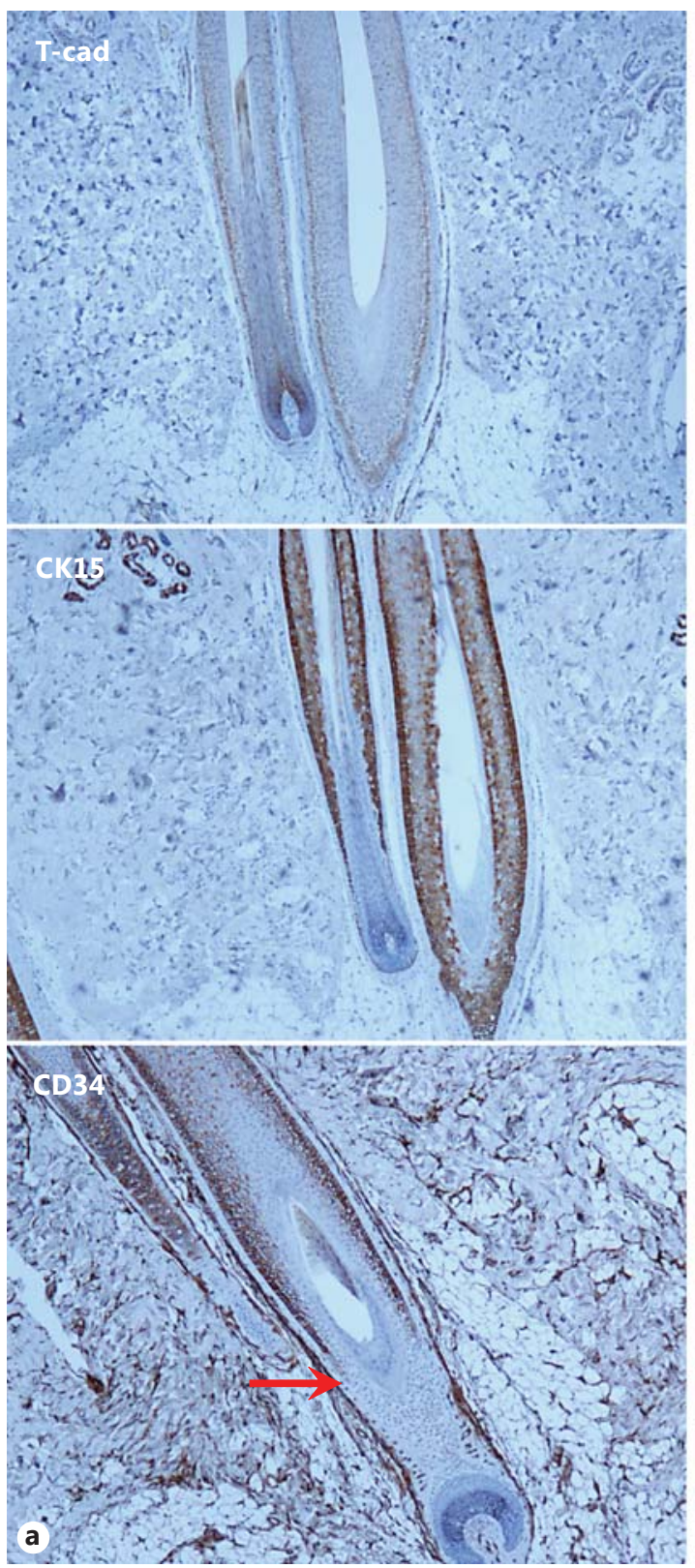

Suprabulbar
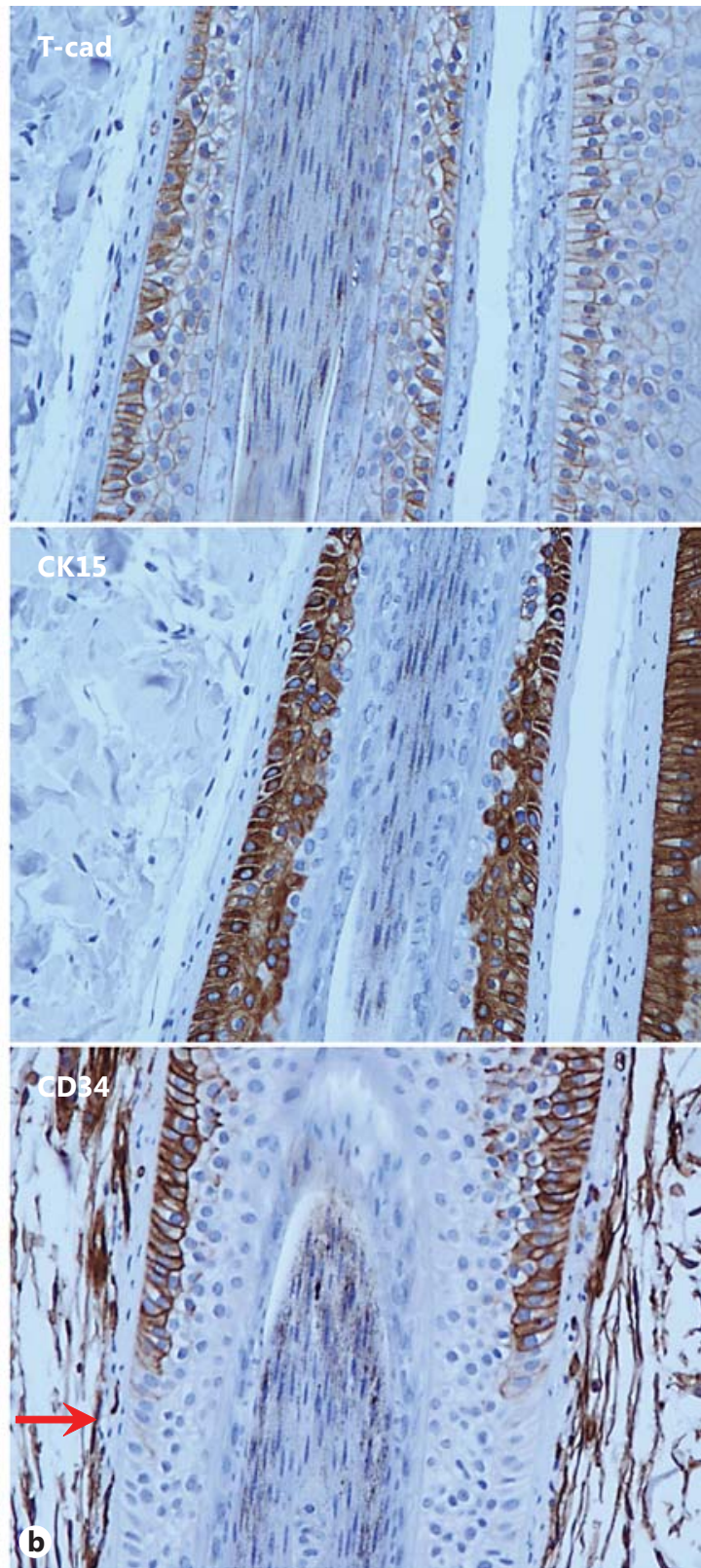

Fig. 6. Expression of T-cadherin, CK15, and CD34 in the suprabulbar region of terminal hair follicles. a Tcadherin, CK15, and CD34 are strongly expressed within the suprabulbar region of the ORS. b High-power image showing strong staining for T-cadherin, CK15, and CD34 in the most external layer of the ORS. Arrow indicates absence of CD34 staining at the lower part of the suprabulbular region.

The function of T-cadherin in skin (patho)biology remains unclear. In vitro investigations exploiting ectopic modification of T-cadherin expression levels in human keratinocyte (HaCaT) and SCC (A431, HSC-1) cell lines have demonstrated functions for T-cadherin as a negative regulator of proliferation, migration, and invasion $[10,11]$ and a positive regulator of cell-matrix adhesion [12]. In keeping with these putative functions, immunohistochemical studies of human cutaneous specimens have reported inverse relationships between 
Buechner et al.: T-Cadherin Expression in the Epidermis and Adnexal Structures of Normal Skin
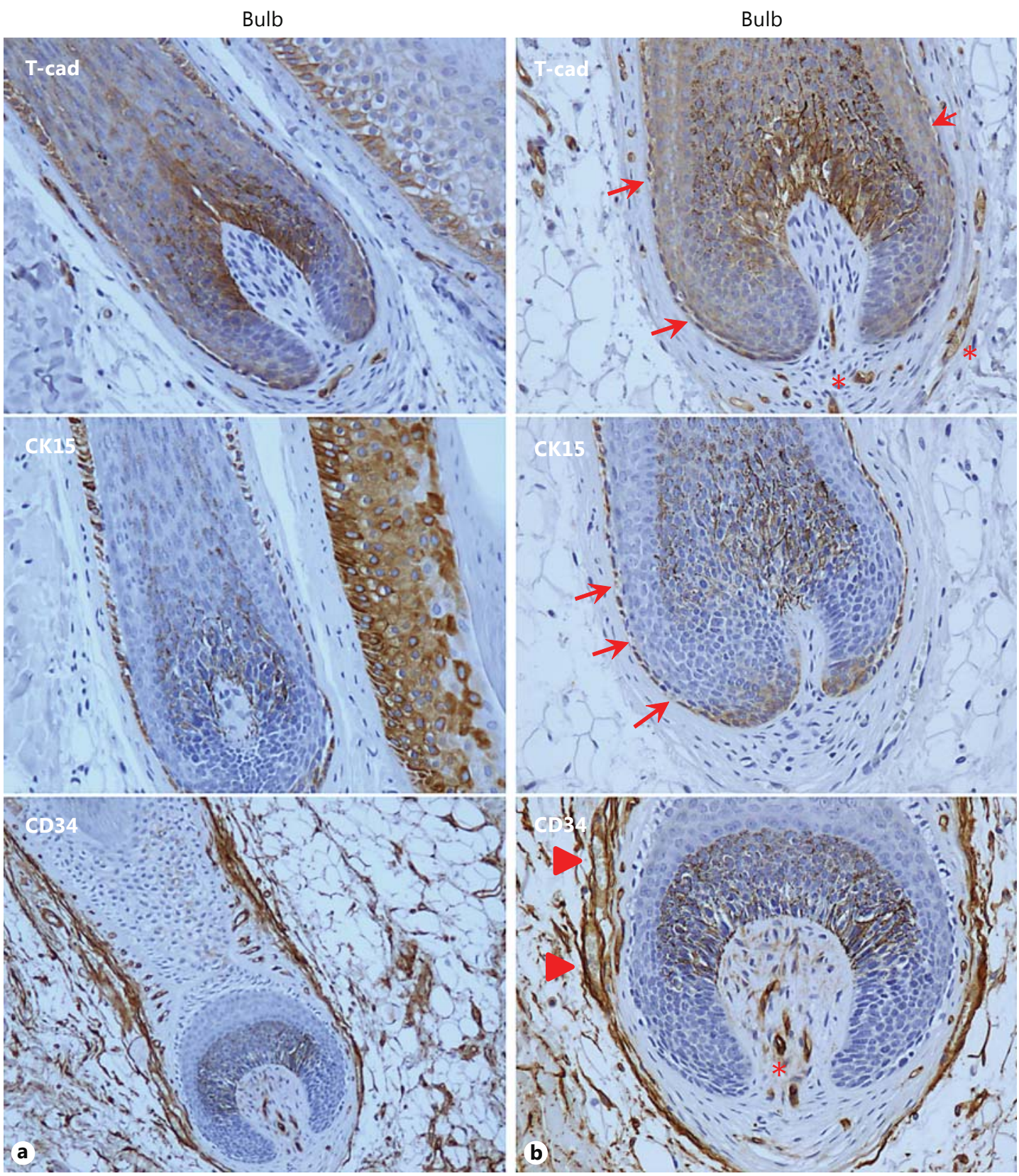

Fig. 7. Expression of T-cadherin, CK15, and CD34 in the bulb region of terminal hair follicles. a T-cadherin and CK15 stain the thin most external layer of the ORS at the bulb level. CD34 expression is not present. b High-power images illustrating that the expression of T-cadherin and CK15 are not positive throughout the ORS (arrows point to some negatively stained cells). Note T-cadherin-positive endothelial cells in the dermal papilla and fibrous root sheath (asterisks). There are CD34-positive endothelial cell (asterisks) and dendritic dermal cells (arrowheads) surrounding the bulb.

T-cadherin expression levels and progression of several hyperproliferative keratinocytic disorders including psoriasis vulgaris [9], Bowen's disease $[6,10]$, and SCC $[6,10]$. On the other hand, and as recapitulated in this study, in the healthy epidermis, T-cadherin is strongly expressed in basal layer keratinocytes [6-8] which actively proliferate. Notably, cells that move away from the basal layer compartment toward the skin surface, withdraw from cell 
cycle, and commit to terminal differentiation no longer express T-cadherin. T-cadherin is also expressed in actinic keratosis $[6,10]$, an in situ SCC characterized by exaggerated keratinocyte proliferation. Furthermore, T-cadherin is prominently upregulated in BCC regardless of grade of proliferation and invasiveness (superficial, nodular, and infiltrative), with expression being particularly strong in the palisading peripheral cells of invading tumor nests [8]. Noteworthy too is that in well-differentiated SCC, T-cadherin remains strongly expressed on cells at the periphery of tumor nests invading the dermis, whereas in moderately-to-poorly differentiated SCC (i.e., transformed cells acquire dedifferentiated status), T-cadherin expression is reduced or lost [10]. Studies using A431 SCC keratinocyte cell line and a threedimensional spheroid cell culture model to mimic multicellular tumor organization demonstrated that spheroid structure was remarkably more compact following ectopic upregulation of T-cadherin but loose following silencing of T-cadherin [10]. These in vitro findings, together with the collective immunohistochemical observations of the specific expression of $\mathrm{T}$-cadherin in the basal keratinocyte layer of the epidermis and its maintained expression in the periphery of BCC and well-differentiated SCC, might be interpreted to indicate an important role for T-cadherin in maintenance of organized structure.

Support for such an interpretation is provided by our findings on the pattern of T-cadherin expression in skin adnexal structures, which, to date, has received rare attention. In sebaceous glands, the basal cell layer exhibited strong expression of T-cadherin, whereas sebocytes in the inner compartment of the gland showed no expression. The latter finding is at variance with Takeuchi et al. [6], who reported expression of T-cadherin in mature sebocytes of sebaceous glands. The expression of T-cadherin in the basal layer of the sebaceous gland is not surprising since the physiological processes of the glands are in many ways similar to those of the continual renewal of the interfollicular epidermis $[13,14]$. Although epidermal terminal differentiation differs from sebocyte maturation, in both the epidermis and sebaceous glands undifferentiated cells of the proliferative basal cell layer are required to maintain the continual renewal of the epidermis as well as the continuous sebum production and regeneration of sebaceous glands $[13,14]$. As for the interfollicular epidermis, basal cells of the sebaceous gland typically lack expression of T-cadherin once they have left the basal compartment, withdrawn from the cell cycle, and commit to terminal sebocyte differentiation.

In normal eccrine glands, we observed positive cytoplasmic staining for T-cadherin in the majority of the inner layer of cells within the secretory coils, confirming the finding of Takeuchi et al. [6]. However, we additionally found that outer layer of myoepithelial cells in the secretory portion and epithelial cells lining the excretory ducts strongly expressed T-cadherin. Pulse and pulse-chase studies using the sensitive nucleotide analog ethynyldeoxyuridine demonstrated proliferation of both luminal and myoepithelial cells within mature coiled secretory sweat glands, supporting that both cell populations are capable of regenerative self-renewal [15]. In contrast to eccrine glands, the inner layer of secretory cells in apocrine sweat glands was consistently negative for T-cadherin, whereas myoepithelial cells within the periphery of the secretory components exhibited positive staining. As for eccrine glands, apocrine gland myoepithelial cells are likely also capable of self-renewal $[14,16]$.

Expression of T-cadherin in the hair follicle, especially in the ORS, has been reported by Takeuchi et al. [5, 6]. Our study clearly demonstrates that expression of T-cadherin in terminal hair follicles is strictly confined to the most external layer of the ORS. It is perhaps not surprising that T-cadherin is expressed throughout the ORS, as the ORS is contiguous with, and biochemically similar to, the basal layer of the epidermis. Epithelial hair follicle stem cells localize to the outermost ORS layer of the distal hair follicle epithelium at the proximal end of the isthmus in the region known as the bulge, and in a cluster of cells below the bulge known as the hair germ $[17,18]$. Using consecutive sections of terminal hair follicles and K15 and 
CD34 antibodies as stem cell markers, we found that both CK15-positive cells in the bulge region and CD34-positive cells in the suprabulbar ORS localize with T-cadherin positivity. Like the basal keratinocyte layer, the bulge and suprabulbular regions of the hair follicle are regarded as important stem cell reservoirs for hair follicle renewal and also for regeneration of sebaceous glands and of the epidermis in case of injury [17-19]. The presence of T-cadherin throughout the bulge and suprabulbular regions might not endow T-cadherin with usefulness in specific hair follicle stem cell identification, but nevertheless invokes potential participation in regenerative processes.

\section{Conclusion}

Accumulating evidence from different fields suggests that T-cadherin acts as a signaling receptor participating in the control of tissue architecture, recognition of the environment, regulation of cell motility, guidance of moving structures, and guarding integrity of functionally connected tissue layers [4]. Herein, we have shown that T-cadherin is expressed in cell layers considered to house regenerative stem cell populations, namely the basal keratinocyte layer of the epidermis, the basal layer of the sebaceous gland, the myoepithelial layer of the eccrine gland, the myoepithelial layer of the apocrine glands, and the hair follicle ORS. A function for T-cadherin as a guardian of the structural integrity of the epidermis and adnexal structures may be crucial to maintain homeostasis of the skin.

\section{Acknowledgements}

Janne Dubler and Barbara Ruch are thanked for their expert assistance in the preparation and staining of tissue sections. Prof. Resink is supported by the Stiftung für Herz- und Kreislaufkrankheiten.

\section{Statement of Ethics}

Skin samples, previously collected for medical diagnostic, were obtained from the archival histopathology collection of the Laboratory for Histologic Diagnostic, Basel. They were treated anonymously.

\section{Disclosure Statement}

The authors have no conflict of interest to declare.

\section{References}

1 Angst BD, Marcozzi C, Magee AI: The cadherin superfamily: diversity in form and function. J Cell Sci 2001;114: 629-641.

2 Brooke MA, Nitoiu D, Kelsell DP: Cell-cell connectivity: desmosomes and disease. J Pathol 2012;226:158-171.

3 Muller EJ, Williamson L, Kolly C, Suter MM: Outside-in signaling through integrins and cadherins: a central mechanism to control epidermal growth and differentiation? J Invest Dermatol 2008;128:501-516.

4 Philippova M, Joshi MB, Kyriakakis E, Pfaff D, Erne P, Resink TJ: A guide and guard: the many faces of T-cadherin. Cell Signal 2009;21:1035-1044. 
5 Takeuchi T, Liang SB, Ohtsuki Y: Downregulation of expression of a novel cadherin molecule, T-cadherin, in basal cell carcinoma of the skin. Mol Carcinog 2002;35:173-179.

6 Takeuchi T, Liang SB, Matsuyoshi N, Zhou S, Miyachi Y, Sonobe H, Ohtsuki Y: Loss of T-cadherin (CDH13, H-cadherin) expression in cutaneous squamous cell carcinoma. Lab Invest 2002;82:1023-1029.

7 Zhou S, Matsuyoshi N, Liang SB, Takeuchi T, Ohtsuki Y, Miyachi Y: Expression of T-cadherin in basal keratinocytes of skin. J Invest Dermatol 2002;118:1080-1084.

-8 Buechner SA, Philippova M, Erne P, Mathys T, Resink TJ: High T-cadherin expression is a feature of basal cell carcinoma. Br J Dermatol 2009;161:199-202.

-9 Zhou S, Matsuyoshi N, Takeuchi T, Ohtsuki Y, Miyachi Y: Reciprocal altered expression of T-cadherin and P-cadherin in psoriasis vulgaris. Br J Dermatol 2003;149:268-273.

$\checkmark 10$ Pfaff D, Philippova M, Buechner SA, Maslova K, Mathys T, Erne P, Resink TJ: T-cadherin loss induces an invasive phenotype in human keratinocytes and squamous cell carcinoma (SCC) cells in vitro and is associated with malignant transformation of cutaneous SCC in vivo. Br J Dermatol 2010;163:353-363.

$\checkmark 11$ Mukoyama Y, Zhou S, Miyachi Y, Matsuyoshi N: T-cadherin negatively regulates the proliferation of cutaneous squamous carcinoma cells. J Invest Dermatol 2005;124:833-838.

12 Mukoyama Y, Utani A, Matsui S, Zhou S, Miyachi Y, Matsuyoshi N: T-cadherin enhances cell-matrix adhesiveness by regulating beta1 integrin trafficking in cutaneous squamous carcinoma cells. Genes Cells 2007;12: 787-796.

13 Niemann C: Differentiation of the sebaceous gland. Dermatoendocrinol 2009;1:64-67.

14 Blanpain C, Fuchs E: Epidermal homeostasis: a balancing act of stem cells in the skin. Nat Rev Mol Cell Biol 2009;10:207-217.

15 Lu CP, Polak L, Rocha AS, Pasolli HA, Chen SC, Sharma N, Blanpain C, Fuchs E: Identification of stem cell populations in sweat glands and ducts reveals roles in homeostasis and wound repair. Cell 2012;150:136-150.

16 Lu C, Fuchs E: Sweat gland progenitors in development, homeostasis, and wound repair. Cold Spring Harb Perspect Med 2014;4:a015222.

17 Hsu YC, Li L, Fuchs E: Emerging interactions between skin stem cells and their niches. Nat Med 2014;20:847856.

18 Purba TS, Haslam IS, Poblet E, Jimenez F, Gandarillas A, Izeta A, Paus R: Human epithelial hair follicle stem cells and their progeny: current state of knowledge, the widening gap in translational research and future challenges. Bioessays 2014;36:513-525.

19 Ohyama M: Hair follicle bulge: a fascinating reservoir of epithelial stem cells. J Dermatol Sci 2007;46:81-89. 